\title{
Intestinal pseudo-obstruction: Rule out systemic lupus erythematous!
}

\author{
Myriam Ayari $^{1}$, Abdelwaheb Nakhli ${ }^{1}$, Zeineb Teyeb ${ }^{1}$, Imen Abdelaali ${ }^{1}$, Syrine Bellakhal ${ }^{1}$, \\ and Taieb Jomni ${ }^{1}$ \\ ${ }^{1}$ La Marsa Internal Security Forces Hospital
}

July 7,2020

\begin{abstract}
Intestinal pseudo-obstruction (IPO) is defined as an intestinal obstruction without mechanical obstructive lesion. It's a rare complication of systemic lupus erythematosus (SLE). We report a case of SLE inaugurated by IPO to emphasize the importance of early recognition of the diagnosis especially that SLE-related IPO responds well to corticosteroid therapy.
\end{abstract}

\section{Key Clinical Message:}

Systemic disease should be always considered when managing unexplained intestinal pseudo-obstruction. Intestinal pseudo-obstruction related to systemic lupus erythematosus is often responsive to corticosteroid therapy when promptly treated.

\section{Introduction:}

Intestinal pseudo-obstruction (IPO) is a gut motility disorder resulting in impairment of intestinal peristalsis leading to severe obstructive symptoms without mechanical causes. Rarely, it can be a manifestation of systemic lupus erythematosus (SLE) [1]. The prevalence of IPO in a series of hospitalized patients with SLE is about $2 \%$ [2]. SLE-related IPO is associated with increased morbidity and mortality, mostly if misdiagnosed and incorrectly treated [2]. We report a case of IPO as first manifestation of SLE to highlight the importance of early recognition of the diagnosis and so avoid unnecessary surgery especially that SLErelated IPO responds well to corticosteroids.

\section{Case presentation:}

A 37-year-old Caucasian female, presented to the emergency room with abdominal pain, distension and vomiting. Symptoms were developed two months ago with reported diarrhea and weigh loss. She already was treated symptomatically but no diagnosis was done and her condition went worse as she developed subacute bowl obstruction signs. She had no other previous significant personal history except polyarthralgia. No concomitant medication was taken. At presentation, physical examination revealed marked abdominal distension, diffuse tympanism with tenderness without rebound tenderness. There was no fever and vital signs were stable. Neurological and cutaneous examinations were normal. Abdominal X-ray imaging showed several air-fluid levels in small and large bowel (figure 1). Investigations blood tests indicated normochromic anemia of $9 \mathrm{~g} / \mathrm{dl}$ with reticulocytes count of $27058 / \mathrm{mm}^{3}$, leucopenia of $3500 / \mathrm{mm}^{3}$, lymphopenia of $500 / \mathrm{mm}^{3}$ and normal platelets count. The blood chemistry showed: hypokaliemia of $2.6 \mathrm{mmol} / \mathrm{L}$, sodium of 142 $\mathrm{mmol} / \mathrm{L}$, albumin of $2.8 \mathrm{~g} / \mathrm{dL}$ and normal lipase level. Thyroid function and hepatic tests were normal and there was no inflammatory syndrome. Haemolysis markers were negative. Dipstick urinalysis showed normal results. Abdominal CT scan revealed dilated loops in the small bowel, segmental thickened small intestinal walls without any mechanical obstacle (figure 2), bilateral pleural, pelvic, abdominal effusion and not marked 
bilateral hydronephrosis. The patient was diagnosed with intestinal pseudo-obstruction and nasogastric tube was placed. Antibiotics against bacterial growth, parenteral nutrition and potassium supplementation were prescribed, as well as intravenous perfusion of erythromycin with moderate improvement of symptoms. Colonoscopy was performed showing segmental thickened and edematous appearance of colonic mucosa with no specific findings at histopathology. Upper endoscopy was normal as well as histological examination of duodenal biopsy. Considering young age, female patient and history of polyarthralgia, we investigated systemic diseases.

Immunological tests showed positive anti-nuclear antibody (ANA) at a titer of 1:400, anti-dsDNA antibodies, anti-SSB and anti-SSA. Direct Coombs' test was positive in the absence of hemolytic anemia.

Thus, she was diagnosed with SLE-related IPO as she met 5 criteria of Systemic Lupus International Collaborating Clinics (SLICC) classification criteria: leucopenia $<4000 / \mathrm{mm}^{3} /$ lymphopenia $<500 / \mathrm{mm}^{3}$, pleural effusion, positive Coombs' test without hemolytic anemia, ANA, anti-dsDNA antibody. Subsequently, we started corticosteroid therapy. The patient received 3 days pulses of methylprednisolone (1000 mg per day) followed by oral prednisolone $1 \mathrm{mg} / \mathrm{kg}$ /day associated with Hydroxychloroquine (400mg/day). Her condition had rapidly improved significantly with disappearance of abdominal symptoms, vomiting and air-fluid levels at $\mathrm{X}$ ray. The patient was discharged four days after starting corticosteroids and was symptom-free by the last time she was reviewed one month later.

\section{Discussion:}

Gastrointestinal involvement is not common among patients presenting SLE [1]. The clinical manifestations may essentially include lupus mesenteric vasculitis, intestinal pseudo-obstruction, protein losing enteropathy, pancreatitis and hepatobiliary manifestations [1]. Intestinal pseudo-obstruction is a gut motility disorder and a rare condition when associated to SLE.

Pathogenesis of SLE-related IPO remains unclear. It can probably be due to intestinal vasculitis affecting visceral smooth muscles or to autoantibodies targeting smooth muscle with immune complex deposition on the muscle and/or nerve explaining gut dismotility disorder during SLE [3].

The diagnosis of IPO is made based on clinical signs, air-fluid levels and bowel dilatation, with or without thickened bowel wall in radiological imaging. Esophagogastroduodenoscopy, colonoscopy, and abdominal CT scan are needed to exclude mechanical obstruction [4]. Intestinal manometry may be needed to exclude systemic sclerosis or Hirschprung's disease [5].

Diagnosis might be challenging especially if IPO is the first manifestation of SLE [3]. Misdiagnosis rate is very high, up to $78 \%$ [2]. However it is important to promptly recognize this rare condition since misdiagnosis can lead to an unnecessary surgical intervention [3].

Ureterohydronephrosis, anti-U1 RNP antibodies, peritonitis and low C3 level were identified as being independent predictors of IPO in SLE [6]. Thus, authors recommend regular abdominal X-ray examinations in patients with these predictors. In our case, hydronephrosis was noticed at first presentation.

Supportive treatments of IPO include parenteral nutrition, prokinetics (neostigmine, erythromycin) and antibiotics against bacterial overgrowth [7]. In addition, specific treatment of SLE-related IPO is based on corticosteroid therapy +/- associated with immunosuppressants (cyclophosphamide / azathioprine / tacrolimus) [3,7]. In a review of the literature published by Wang et al, 27 patients received corticosteroids as initial therapy. A good response was obtained in $81 \%$ of the cases [3]. However, none of the patients who underwent surgical intervention achieved long-term improvement [3,7]. In addition to ineffectiveness, unnecessary surgical intervention is responsible of morbi-mortality. However, urgent surgery must be indicated in acute complications of IPO such as intestinal perforation or ischemia. Regarding prognostic, IPO-related SLE can cause significant morbidity and mortality if not rapidly diagnosed and treated. In another review of the literature [7], mortality rate was of $6.99 \%$ (10/143). Nephrotic syndrome, ureterohydronephrosis and megacholedochus are independent poor prognostic factors in SLE-related IPO [2]. In this report, although the patient had hydronephrosis, she presented favorable outcome. 
It is important to timely initiate medical treatment, otherwise the smooth muscle layer can progress to fibrosis, become atrophic and no longer reversible [8].

We reported a case of IPO presenting as the initial manifestation of systemic lupus erythematosus. The diagnosis of IPO was based on clinical signs, X-ray, CT scan and endoscopy. The diagnosis of SLE was made upon clinical, biological criteria and immunological findings. The patient had a spectacular response to steroids. Treatment with corticosteroids alone was sufficient and the patient did not require other immunosuppressive therapy.

In conclusion, SLE-related IPO is rare and may be a challenging diagnosis especially if the underlying disease is not yet established. Systemic disease should be always considered when managing unexplained IPO, as medical treatment based on corticosteroids +/- immunosuppressive therapy is very efficient.

\section{Conflict of interest:}

None.

\section{Patient's Consent:}

Yes.

\section{Author Contributions:}

All authors participated in the clinical care of the patient. Myriam Ayari and Abdelwaheb Nakhli designed the concept of the manuscript, were involved in the definition of intellectual content and in the literature search. Myriam Ayari, Abdelwaheb Nakhli, Syrine Bellakhal, Zeineb Teyeb and Taieb Jomni were involved in the manuscript preparation. Taieb Jomni, Syrine Bellakhal, Imen Abdelaali contributed to the critical review of the manuscript. Taieb Jomni acted as Guarantor for the research.

\section{References}

[1] Brewer BN, Kamen DL. 2018. Gastrointestinal and Hepatic Disease in Systemic Lupus Erythematosus. Rheum Dis Clin North Am 44:165-75.

[2] Zhang L, Xu D, Yang H, Tian X, Wang Q, Hou Y, et al. 2016. Clinical Features, Morbidity, and Risk Factors of Intestinal Pseudo-obstruction in Systemic Lupus Erythematosus: A Retrospective Case-control Study. J Rheumatol 43:559-64.

[3] Wang J, Liu G, Liu T, Wei J. 2014. Intestinal Pseudo-Obstruction in Systemic Lupus Erythematosus: A Case Report and Review of the Literature. Medicine (Baltimore) 93:E248.

[4] Oh DJ, Yang JN, Lim YJ, Kang JH, Park JH, Kim MY. 2015. Intestinal Pseudo-Obstruction as an Initial Manifestation of Systemic Lupus Erythematosus. Intest Res 13:282-6.

[5] Cogliandro RF, De Giorgio R, Barbara G, Cogliandro L, Concordia A, Corinaldesi R, et al. 2007. Chronic intestinal pseudo-obstruction. Best Pract Res Clin Gastroenterol 21:657-69.

[6] Huang Q, Lai W, Yuan C, Shen S, Cui D, Zhao J, et al. 2016. Predictors of intestinal pseudo-obstruction in systemic lupus erythematosus complicated by digestive manifestations: data from a Southern China lupus cohort. Lupus 25:248-54.

[7] Li Z, Xu D, Wang Z, Wang Y, Zhang S, Li M, et al. 2017. Gastrointestinal system involvement in systemic lupus erythematosus. Lupus 26:1127-38.

[8] Park FD, Lee JK, Madduri GD, Ghosh P. 2009. Generalized megaviscera of lupus: refractory intestinal pseudo-obstruction, ureterohydronephrosis and megacholedochus. World J Gastroenterol 15:3555-9.

List of figure legends:

Figure 1: $\mathrm{X}$ ray image at presentation showing several air-fluid levels 
Figure 2: Abdominal CT scan views showing large dilated small bowel and thickened small intestinal wall

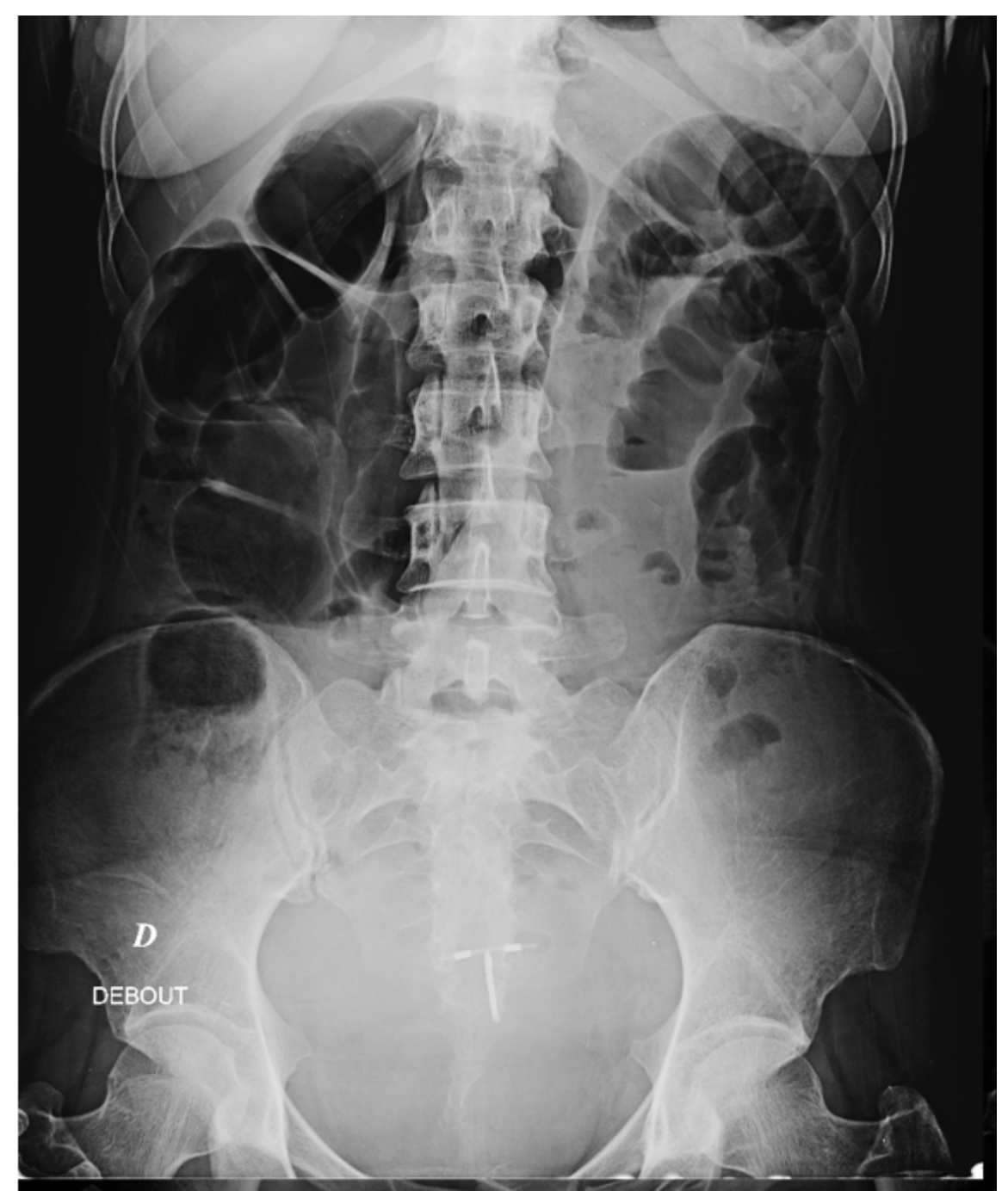



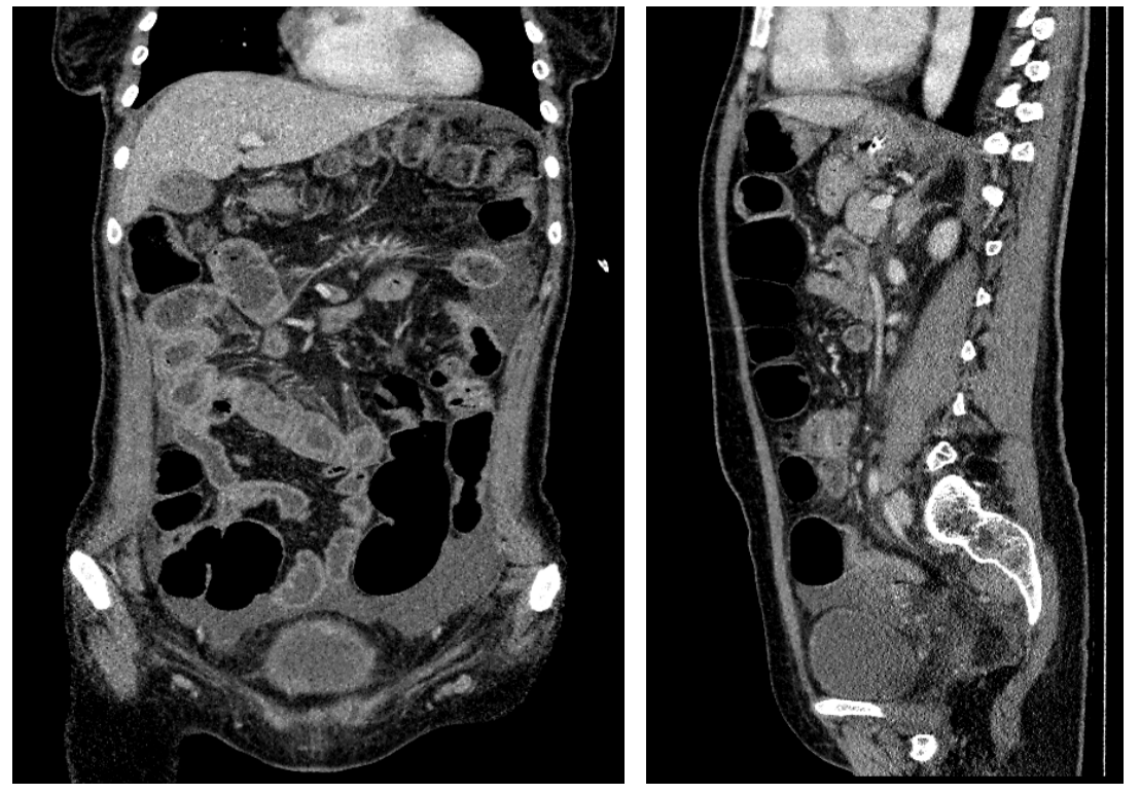